\title{
Large enhancement of emergent magnetic fields in MnSi with impurities and pressure
}

\author{
Benjamin J. Chapman, ${ }^{1}$ Maxwell G. Grossnickle ${ }^{\dagger},{ }^{1}$ Thomas Wolf, ${ }^{2}$ and Minhyea Lee ${ }^{1, *}$ \\ ${ }^{1}$ Department of Physics, University of Colorado, Boulder, CO 80309, USA \\ ${ }^{2}$ Institute for Solid-State Physics, Karlsruhe Institute of Technology, D-76021 Karlsruhe, Karlsruhe, Germany
}

(Dated: October 18, 2018)

\begin{abstract}
We report a study of the topological Hall effect (THE) in Fe-doped MnSi and compare with results from pure $\mathrm{MnSi}$ under pressure. We find that Fe doping increases the THE, indicating an enhancement of the magnitude of the emergent gauge field. This is consistent with the concurrent reduction in the length scale of the skyrmion lattice. For both pressurized and doped samples, we calculate the emergent magnetic field based on the size of the measured THE, and compare it with a theoretical upper-bound. We find that the ratio of these two remains more or less constant with pressure or Fe doping, but differs greatly from that of pure MnSi at ambient pressure. We discuss the implications of this ratio with respect to trends in the saturated magnetic moment and helical pitch length as $T_{C} \rightarrow 0$ via doping and pressure, respectively.
\end{abstract}

PACS numbers:

A new type of magnetically-ordered state was recently discovered [ $1[-5]$ to form a lattice from vortex-like objects in the spin texture, so-called skyrmions [6]. Twodimensional skyrmion lattices (SLs) are stabilized in the presence of an external magnetic field and thermal fluctuations near the Curie temperature $\left(T_{C}\right)$, occupying a small pocket in the temperature $(T)$ - applied magnetic field $(B)$ phase diagram (the $A$-phase). They orient perpendicular to the applied field $B \hat{z}$, and have been observed with small angle neutron scattering (SANS) [1, 2] and Lorentz tunneling electron microscopy [3 [5], exclusively in B20-structures with cubic symmetry (space group $P 2_{1} 3$ ). Celebrated examples include the itinerant ferromagnet $\mathrm{MnSi}$ [1], semiconductors $\mathrm{Fe}_{x} \mathrm{Co}_{1-x} \mathrm{Si}$ [2, 5] and $\mathrm{FeGe}$ 3], and insulator $\mathrm{Cu}_{2} \mathrm{SeO}_{3}$ 4, 7]. The $\mathrm{B} 20$ crystal structure lacks inversion symmetry, allowing a new energy scale $(D)$, the Dzyaloshinsky-Moriya interaction [8, 9], to compete with the conventional exchange interaction $(J)$. This results in a helically ordered magnetic ground state with well defined pitch $\lambda$, whose length is proportional to $J / D$. The length scale for the unit cell of an SL is also set by $\lambda$ [10], which runs from a few $\mathrm{nm}$ to a few tens of nm, depending on the compound.

The non-coplanarity of spin structures like hexagonal SLs can give rise to an emergent magnetic field $\boldsymbol{b}_{r}$ expressed [11, 12] as

$$
b_{r}^{i}=\frac{\Phi_{0}}{8 \pi} \epsilon_{i j k} \hat{n} \cdot\left(\partial_{j} \hat{n} \times \partial_{k} \hat{n}\right) .
$$

Here $\epsilon_{i j k}$ is the Levi-Civita symbol whose indices run over $x, y$, and $z, \hat{n}(\boldsymbol{r})$ is a unit vector of the magnetization $\boldsymbol{M}(\boldsymbol{r})$, and $\Phi_{0}=h /|e|$ is the single-electron flux quantum. The hexagonal SL in MnSi is uniform in the $\hat{z}$ direction, so only the $z$-component of $\boldsymbol{b}_{\boldsymbol{r}}$ is non-vanishing: $\boldsymbol{b}_{\boldsymbol{r}}=\left(0,0, b_{r}\right)$. This field arises in the strong Hund coupling limit, where the spin of conduction electrons orients parallel to the local magnetization, twisting to follow it as they move through the material. This results in the acquisition of an extra phase factor in their wave functions, represented by the line integral of a vector potential, analogous to the Aharonov-Bohm effect [13]. The curl of that vector potential is the quantity given in Eq. 1. which acts on conduction electrons in a similar way as the physical magnetic field [14, 15], and causes the topological Hall effect (THE).

Similarly, the intrinsic anomalous Hall effect in ferromagnets is caused by a fictitious k-space magnetic field $\boldsymbol{b}_{k}$ (see [16] and references therein) that arises from a Berry-curvature in momentum space. In fact, it was recently suggested [17] that chiral magnets playing host to SLs may be the first experimental examples of systems in which mixed-Berry phases, that is, Berry phases acquired from closed orbits in the full 6-dimensional phase-space $(\boldsymbol{r}, \boldsymbol{p})$, may play an important role.

In an SL, the area integral of $\boldsymbol{b}_{\boldsymbol{r}}$ over a magnetic unit cell is quantized to an integer times $\Phi_{0}[11,18]$. For $\mathrm{MnSi}$ that integer is -1 [1], and the similarity in SANS data of $8 \% \mathrm{Fe}$ doped samples [10] suggests this topological quantum number is unchanged with the level of doping considered here:

$$
\oint_{\mathcal{A}_{s k}} \boldsymbol{b}_{\boldsymbol{r}} \cdot \mathrm{d} \mathcal{A}=-\Phi_{0}
$$

where $\mathcal{A}_{s k}=(2 / \sqrt{3}) \lambda^{2}$ is the area of the magnetic unit cell and the minus sign implies that the emergent field on average opposes the normal vector of the unit cell, which is parallel to the applied $B$. Eq. 2 allows estimation of an upper bound for the average topological magnetic field induced by the spin texture: $\overline{b_{r}}=-\Phi_{0} / \mathcal{A}_{s k}$. The size of this field is tunable via control of $\lambda$ and typically tens of Tesla in $\mathrm{Mn}_{1-x} \mathrm{Fe}_{x} \mathrm{Si}$ (See Table 【).

Hall effects arising from non-trivial spin textures 21, 25] provide a rare opportunity to directly access gauge fields of purely quantum-mechanical origin. To connect $\overline{b_{r}}$ to our experimental results, we introduce a ratio $f=$ $B_{\text {eff }} / \overline{b_{r}}$ between the theoretial upper bound $b_{r}$ and an 


\begin{tabular}{l||r||r|r|r|r|r|r}
\hline & $0 \%$ & $8 \mathrm{kbar}$ & $6 \% \mathrm{Fe}$ & $10 \mathrm{kbar}$ & $9 \% \mathrm{Fe}$ & $13 \mathrm{kbar}$ & $13 \% \mathrm{Fe}$ \\
\hline$T_{C}[\mathrm{~K}]$ & $29.5 \pm 0.2$ & $17.8 \pm 0.3$ & $14.5 \pm 0.6$ & $13.4 \pm 0.2$ & $8.5 \pm 0.25$ & $7.3 \pm 0.1$ & $5.5 \pm 0.3$ \\
$m_{s}\left[\mu_{B}\right][19]$ & $0.43 \pm 0.05$ & $0.36 \pm 0.2$ & $0.31 \pm 0.05$ & $0.3 \pm 0.1$ & $0.22 \pm 0.05$ & $0.31 \pm 0.1$ & $0.19 \pm 0.05$ \\
\hline \hline$R_{H}[\mathrm{n} \Omega \mathrm{cm} / \mathrm{T}]$ & $+7.3 \pm 0.1[20]$ & $+9.6 \pm 1.6$ & & $+7.4 \pm 1.5$ & $-2.0 \pm 0.7$ & $+5.0 \pm 0.5$ & $-1.0 \pm 0.7$ \\
$\rho_{y x}^{T} \max [\mathrm{n} \Omega \mathrm{cm}]$ & $-4.5 \pm 1[21]$ & $-38 \pm 2$ & $+7 \pm 1$ & $-43.7 \pm 1.7$ & $+20 \pm 1$ & $-31.4 \pm 2.2$ & $32 \pm 2$ \\
$\lambda[\mathrm{nm}][22,23]$ & $17.4 \pm 0.4$ & $15.2 \pm 0.2$ & $12.4 \pm 0.4$ & $15.1 \pm 0.2$ & $10.4 \pm 0.4$ & $14.4 \pm 0.1$ & $7.4 \pm 0.5$ \\
\hline$B_{\mathrm{eff}}=\rho_{y x}^{T} / R_{H}[\mathrm{~T}]$ & $-0.61 \pm 0.14$ & $-3.9 \pm 0.7$ & & $-5.9 \pm 1.3$ & $-10.0 \pm 3.5$ & $-6.3 \pm 0.8$ & $-30 \pm 21$ \\
$\overline{b_{r}}=\Phi_{0} / \mathcal{A}_{s k}[\mathrm{~T}]$ & $-11.9 \pm 0.4{ }^{a}$ & $-15.5 \pm 0.2$ & $-23.1 \pm 0.9$ & $-15.7 \pm 0.2$ & $-33.3 \pm 1.7$ & $-17.1 \pm 0.1$ & $-66 \pm 6$ \\
$f=B_{\mathrm{eff}} / \overline{b_{r}}$ & $0.05 \pm 0.01$ & $0.25 \pm 0.04$ & & $0.38 \pm 0.08$ & $0.30 \pm 0.11$ & $0.36 \pm 0.04$ & $0.45 \pm 0.32$ \\
\hline
\end{tabular}

TABLE I: The effects of Fe doping and pressure on the emergent magnetic fields in MnSi. Values for $\rho_{y x}^{T}$ are the maximum topological signal from $B$ sweeps. $R_{H}$ s were computed with fits to Eq. 3 for $0 \%, 9 \%$, and $13 \%$ Fe. For MnSi under $P$ they were extracted by computing the slope of $\rho_{y x}$ when $B>B_{p}$.

${ }^{a}$ This was estimated as $13.15 \mathrm{~T}$ in other work [24], the discrepany arises from use of a slightly different $\lambda$ in $b_{r}$ 's calculation.

effective field $B_{\text {eff }}$ estimated from the magnitude of THE and the normal Hall coefficient $R_{H}$, viz. $\rho_{y x}^{T}=R_{H} B_{\text {eff }}$ [14].

Motivated to tune the skyrmion size set by $\lambda$, and thus control the magnitude of $\overline{b_{r}}$ we studied the Hall effect in Fe doped MnSi. The primary effect of increasing Fe is a suppression of $T_{C}$ and the saturated magnetic moment $\left(m_{s}\right)$ [26, 27], both of which monotonically go to zero, vanishing at the critical doping $x_{c} \simeq 15-19 \%$ [22, 27]. Previous studies have shown that Fe doping leads to a linear decrease in $J$ but leaves $D$ unchanged [22], resulting in a decrease in $\lambda$ observed in scattering experiments 10, 22]. This compresses the spatial extent of the skyrmions, squeezing their single flux quantum through a smaller area, and necessitating a larger $\overline{b_{r}}$.

To provide insight on how the suppression of $T_{C}$ affects emergent magnetic fields, we compare the above results with Hall measurements of pure MnSi under pressure [17, 25]. Like Fe doping, pressure $(P)$ also suppresses $T_{C}$, reaching zero at the critical pressure $P_{C} \simeq 15 \mathrm{kbar}$. $m_{S}$, however, is not as severely suppressed as in doped samples with comparable $T_{C} \mathrm{~s}$, and even remains non-zero at $P_{C}$ [19]. Furthermore, $\lambda$ is reduced only slightly under pressure: at $P_{C}, \lambda$ is $\approx 80 \%$ of its length at ambient pressure $\left(\lambda_{0}\right)$ 23]; it falls to $40 \%$ of $\lambda_{0}$ with $13 \%$ Fe doping. We return to these differences in later comparisons of the THE.

We find that the topological Hall effect in Fe doped samples is enhanced by factors of 2,5 , and 8 with respect to MnSi. The sign of the THE flips as well, remaining opposite to the sign of $R_{H}$, in accordance with the minus sign in Eq. 2. $f=B_{\text {eff }} / \overline{b_{r}}$ ranges between 0.25 and 0.45 for doped and pressurized samples, while for $\mathrm{MnSi}$ at ambient $P$ the ratio is much lower, only 0.05 . We discuss later the implications of these disparate $f$ values. Table凹 summarizes our experimental results and calculations of the emergent fields in $\mathrm{MnSi}$ subjected to $\mathrm{Fe}$ doping and $P$.
Single crystals of $\mathrm{MnSi}$ and $\mathrm{Mn}_{x} \mathrm{Fe}_{1-x} \mathrm{Si}$ were grown by the Bridgman technique, and cut with typical dimensions $\sim 2 \mathrm{~mm} \times 0.7 \mathrm{~mm} \times 100 \mu \mathrm{m}$. Six contacts of gold wire and silver paint were used for Hall and magnetoresistance measurements, for which DC currents of $0.5-4 \mathrm{~mA}$ were applied. All data presented here are for experiments with $B$ along the $\langle 111\rangle$ direction, with the current perpendicular to $B$, and demagnetization effects corrected according to Ref. 28]. Typical residual resistivity ratios, determined by $\rho(300 \mathrm{~K}) / \rho(2 \mathrm{~K})$, were $80,8,5$, and 4 for the $0 \%, 6 \%, 9 \%$, and $13 \% \mathrm{Fe}$ contents. Methods for the pressure experiments are described in [25].

Fig. 1(c) shows the $T$ dependence of the longitudinal resistivity $\rho$ at zero field. $T_{C}$ S of $29.5,14.5,8.5$, and $5.5 \mathrm{~K}$ were determined from the discontinuity in $\mathrm{d} \rho / \mathrm{d} T$, consistent with $M$ vs $T$ (data not shown) for $0 \%, 6 \%$, $9 \%$, and $13 \%$ respectively. In general, higher Fe contents are more resistive. Reduction of $m_{S}$ upon Fe doping is also obvious in Fig. 1(b), which shows $M$ as a function of $B$ taken at different fixed $T \mathrm{~s}$. Panel (a) of Fig. 1 shows the resistivities normalized by $\rho\left(T=T_{C}\right)$, as a function of $T / T_{C}$. Note the obvious trend: as $T_{C}$ is suppressed by pressure or doping, the fractional change in resistivity is less sensitive to fractional changes in temperature.

The transverse resistivity $\rho_{y x}$ is plotted as a function of $B$ in Fig. 2. (a-c) show 6\%, 9\%, and $13 \%$ Fe content. For purposes of comparison, (d-f) show pure MnSi at 8, 10, and $13 \mathrm{kbar}$, pressures chosen to have comparable $T_{C} \mathrm{~s}$ to the doped samples. Note the $y$-axes' scales are different for each panel, with the bar indicating $20 \mathrm{n} \Omega \mathrm{cm}$. The unique field profile of $\rho_{y x}$ near $T_{C}$ clearly demonstrates a topological contribution $\rho_{y x}^{T}$, visible as a bulge within the narrow range $0.2<B<0.35 \mathrm{~T}$ in both $6 \%$ and $9 \%$, and broadening in the $13 \%$ data. The maximum values of $\rho_{y x}^{T}$ for $6 \%, 9 \%$ and $13 \%$ are about 2,5 and 8 times bigger than the signal reported in $\mathrm{MnSi}$ at ambient $P$ 21], consistent with an increasing $\overline{b_{r}}$ caused by a reduction of the magnetic unit cell's size. The sign of $\rho_{y x}^{T}$ is 


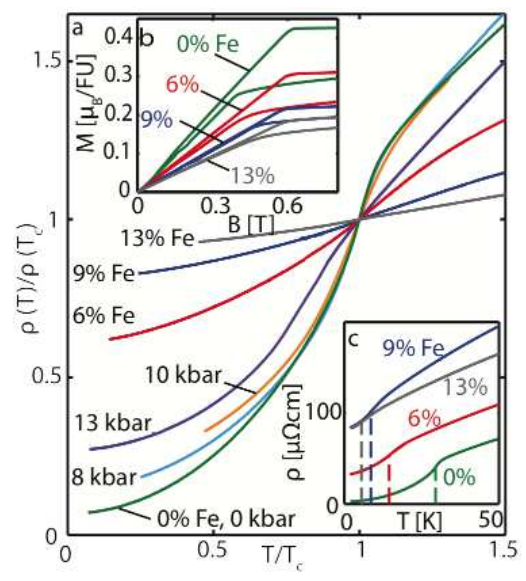

FIG. 1: (a): Normalized resistivities $\rho(T) / \rho\left(T=T_{C}\right)$ as a function of $T / T_{C}$ for both $\mathrm{Mn}_{1-x} \mathrm{Fe}_{x} \mathrm{Si}$ and $\mathrm{MnSi}$ under $P$. (b): $M-B$ curves for the doped samples $2 \mathrm{~K}$ and $T_{C}$. (c): $\rho(T)$ for $0 \%, 6 \%, 9 \%$, and $13 \%$, for which $T_{C}$ s are $29.5 \mathrm{~K}, 14.5 \mathrm{~K}$, $8.5 \mathrm{~K}$, and $5.5 \mathrm{~K}$ (vertical lines). For MnSi under $P, \rho(T)$ is of comparable magnitude to the $0 \%$ Fe curve in (b).

positive for all doped samples. This can be contrasted with MnSi under $P$ (see Table $\llbracket$ ), where THE is always negative and of even larger magnitude, consistent with previous reports [17, 25].

While pure $\mathrm{MnSi}$ displays hole-like carriers (i.e. a positive $R_{H}$ ) up to $200 \mathrm{~K}$ [20], $9 \%$ Fe substitution flips the sign of $R_{H}$, implying the majority carriers are electronlike below $20 \mathrm{~K}$. This is consistent with a simple band picture where $n_{\mathrm{eff}}=1 /\left(R_{H}|e|\right)$, and addition of electrons via Fe doping changes the majority carrier type. Similar behavior is observed in the $13 \%$ sample. Both recover the positive linear $B$ dependence of $\rho_{y x}$ at $T \gg T_{C}$, implying the dominance of positive extrinsic carriers at high $T$.

On the other hand, the Hall signal in $6 \%$ exhibits a complex dependence on $T$ and $B$, which makes its division into the normal, anomalous, and topological Hall effects challenging. Detailed $\rho_{y x}$ traces for the $6 \%$ sample are shown in the supplementary information.

We observe that in addition to an increase of the topological Hall effect, substitution of Fe also increases the anomalous Hall effect (AHE), consistent with the concurrent increase of the longitudinal resistivity $\rho$. The sign of the AHE changes with Fe content as well: it is negative for $0 \%$ [20] and $6 \%$, and positive for $9 \%$ and $13 \%$ (see supplementary information). When $T \ll T_{C}, \rho_{y x}$ varies in direct accordance with $M(B)$ (see Fig. 1 (b)), which implies the AHE dominates in this regime. The clear kink at low $T$ s marks the spin alignment field $B_{p} \approx 0.6$ $\mathrm{T}$ at which all spins become collinear, forcing $b_{r}$ to be 0 . At intermediate $T$, the normal, anomalous, and topological Hall effects all contribute to $\rho_{y x}$, and it is in general non-trivial to distinguish them, which we discuss shortly.

The striking differences in $\rho_{y x}$ between $P$ and doping are (i) The AHE is significantly smaller under $P$, consistent with the reduction in $\rho$. (ii) The THE is larger under $P$, relative to doped samples with comparable $T_{C} \mathrm{~s}$. This is sensible, given that $m_{s}$ values are higher under pressure than in comparable doped samples, and a larger $m_{s}$ increases the overall Hund interaction between the spin texture and conduction electrons. (iii) For MnSi under $P$, the region in the $T-B$ plane where the THE appears extends to lower $T$ s than in comparable doped samples. Still, a similar broadening in the $B$ dimension occurs both in the $13 \% \mathrm{Fe}$ doped sample and in pure $\mathrm{MnSi}$ at $13 \mathrm{kbar}$ (see Fig. 2(c),(f) and Ref. 17]). Such an extension has been observed in 2D FeGe films [3], where low dimensionality enhances fluctuations, and helps stabilize the spin texture in a larger range of $T$ and $B$. In our system, suppression of $T_{C}$ may play a similar role.

To parse the different Hall effects in $\mathrm{Mn}_{1-x} \mathrm{Fe}_{x} \mathrm{Si}$, we combined measurements of the magnetoresistance (MR) and $M$ over field sweeps of \pm 5 T. For $9 \%$ and $13 \%$, this allowed fittings of $\rho_{y x}$ with the expression

$$
\rho_{y x}(B, T)=R_{H} B+S_{H} \rho^{2} M\left(1+\alpha \frac{\rho_{0}}{\rho}\right)+\rho_{y x}^{T},
$$

as in Ref. [20]. Here $R_{H}$ and $S_{H}$ are the normal and anomalous Hall coefficients, and the fitting parameters are $R_{H}, S_{H}$, and $\alpha . \rho_{0}$ denotes $\rho(B=0)$. The first two terms correspond to $\rho_{y x}^{N}$ and $\rho_{y x}^{A}$. Note that the second term $\rho_{y x}^{A}$ contains both intrinsic contributions to the AHE, where the anomalous Hall conductivity $\sigma_{x y}^{A}$ is linearly proportional to $M$ [20], as well as extrinsic contributions to the AHE, where $\rho_{y x} \propto \rho[16,29]$. The fitting parameter $\alpha$ characterizes this proportionality, which was found to be zero for MnSi [20].

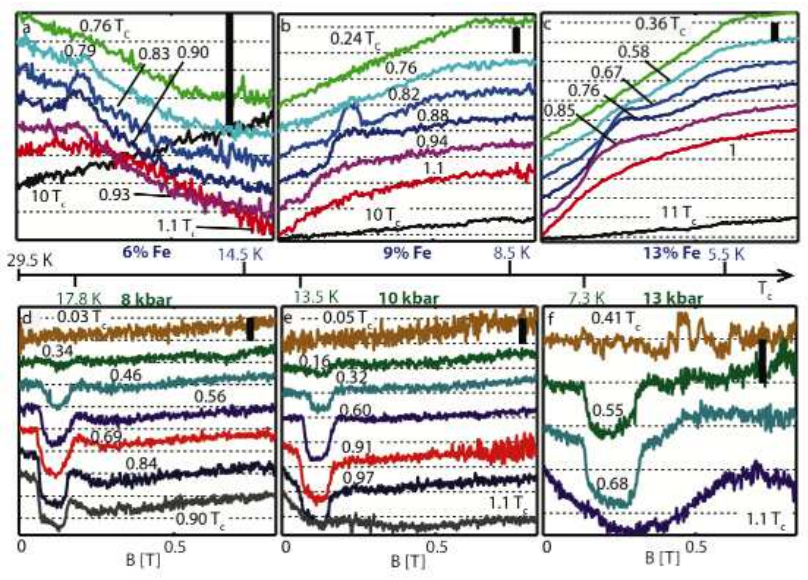

FIG. 2: $\rho_{y x}$ as a function of $B$ for $\mathrm{Mn}_{1-x} \mathrm{Fe}_{x} \mathrm{Si}$ (a)-(c) and $\mathrm{MnSi}$ under pressure (d)-(f). Black bars indicate scale of 20 $\mathrm{n} \Omega \mathrm{cm}$. Different traces correspond to different fixed $T \mathrm{~s}$ in units of $T_{C}$. The temperature scale in the center shows how pressure and doping suppress $T_{C}$ for each dataset. Curves are offset for readability. 
The topological contribution $\rho_{y x}^{T}$ is obtained by subtracting $\rho_{y x}^{N}$ and $\rho_{y x}^{A}$ (estimated from fitting) from the measured $\rho_{y x}$ shown in Fig. 2. For this reason our fitting results are focused on the $A$-phase. To estimate the topological signal for $6 \%$, where the Hall signal is not described by Eq. 3 (see supplementary information), a linear background was subtracted from the Hall traces, as in [17, 21]. The pressure data were analyzed in the same way.

Table \ summarizes our results on the THE, calculations of the emergent magnetic field $B_{\text {eff }}$, and comparison with theoretical upper bounds, for both Fe doping and $P$. The areas used to calculate $\overline{b_{r}}$ were extracted from interpolations of the helical wave-vector measured with neutron scattering on samples of nearby Fe content [22]. $B_{\text {eff }}$, calculated from the measured $\rho_{y x}^{T}$ and $R_{H}$, is expected to be always smaller than $\overline{b_{r}}$ (a theoretical upperbound) such that $B_{\text {eff }}=f \overline{b_{r}}$ with $0<f<1$, and this is observed. Interestingly, despite the larger size of the THE in MnSi under $P, f$ remains more or less constant across both doping and pressure. We suggest three physical considerations that will be reflected in the value of $f$ : (i) The strength of the coupling between charge carriers and the spin texture. Only in the strong Hund coupling limit, where the spin of conduction electrons tightly follows the spin-texture, can $f$ approach unity. (ii) The ratio of conduction electrons with majority to minority spins. This can be varied by changes in the band structure through addition of Fe. Such alterations change the magnitude of the THE, as electrons with opposite spin feel opposite emergent fields. These induce opposite Hall voltages which cancel with one another. (iii) The presence of strong fluctuations in the spin texture on much shorter 30] time scales than our measurement (ms). This is expected to reduce the time-averaged value of the emergent field $B_{\text {eff }}$, and hence diminish $f$.

Another observation from our study on Fe doped MnSi is the identification of a positive MR which is prominent against a background of negative MR only for temperatures and magnetic fields where the THE appears. This clearly shows that longitudinal electric transport is also sensitive to the presence or absence of SLs in $\mathrm{Mn}_{1-x} \mathrm{Fe}_{x} \mathrm{Si}$.

Fig. 3 (a)-(c) shows the magnetoresistance at various fixed $T \mathrm{~s}$. Traces at $2 \mathrm{~K}$ indicate a clear kink at $B_{p}$, attributed to a reduction of spin scattering in the transition from a conical to spin-collinear configuration. As $T$ increases, thermal fluctuations decrease the efficacy of the Zeeman coupling, and $B_{p}$ decreases slightly.

As $T$ approaches $T_{C}$, however, the $B$ profile of the MR changes to yield a positive $\mathrm{MR}$ within a narrow range of $B$, which coincides well with the range of $B$ where the THE appears. The feature is directly visible in the $9 \%$ and $13 \%$ traces in Fig. 3. as $T$ approaches $T_{C}$ from below, a horn-like feature begins to emerge, then becomes
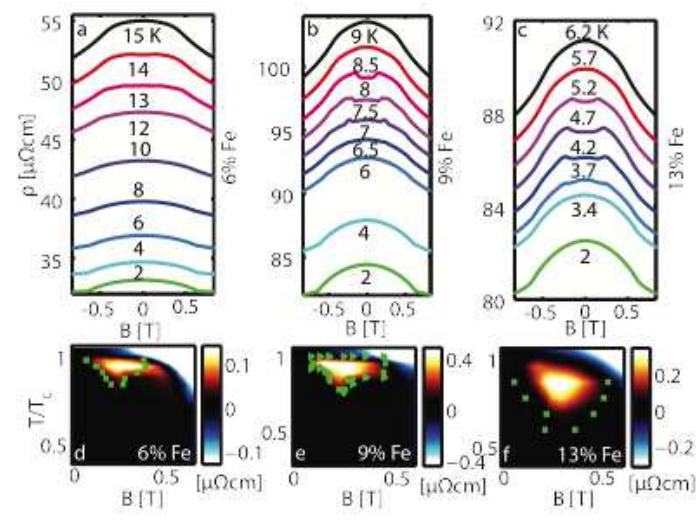

FIG. 3: (a)-(c): Magnetoresistance $\rho_{x x}=\rho$ at fixed temperatures. (d)-(f): MR after subtracting the background from the conical phase (see text). The difference is non-zero only in the $A$-phase, the boundaries of which are confirmed by the presence of the THE in sweeps of $T$ (triangles and circles) and $B$ (squares).

wider as $T$ increases. It vanishes and the MR recovers a smooth and monotonically decreasing dependence on $B$ when $T>T_{C}$. We note that a similar horn-like feature, which disappears above $T_{C}$, was also observed in $\mathrm{MnSi}$ under $P$ [17, 25].

To make the feature more discernible, we empirically estimate the background of the spin scattering reduction to be proportional to $B^{2}$, thus the horn-like feature $\Delta \rho^{T}(B)$ is estimated as $\Delta \rho^{T}(B)=\rho(B)-\left[\rho_{0}-a B^{2}\right]$, where $\rho_{0}=\rho(B=0)$ and $a$ is a positive and weakly $T$-dependent fitting parameter.

In Fig. 3 (d)-(f), we plot $\Delta \rho^{T}$ in the $T$ - $B$ plane with the magnitude of $\Delta \rho^{T}$ indicated by the color scale. The plots delineate a region in the $T-B$ plane which agrees with the boundaries set by the THE, in sweeps of both $T$ (triangles, warming; circles, cooling) and $B$ (squares).

In summary, measurements of the THE in $\mathrm{Mn}_{1-x} \mathrm{Fe}_{x} \mathrm{Si}$ have revealed the tens-of-Tesla emergent magnetic fields generated by its exotic spin texture, and showed they can be enhanced in a controlled manner with Fe doping. As $T_{C}$ is suppressed with increasing $x$, we observe changes in the sign and magnitude of the THE, consistent with an enhancement of the gauge field by the reduction of the magnetic unit cell and the sign change of $R_{H}$. Moreover, a positive $\mathrm{MR}$ in Fe doped samples was observed only within a narrow region of the $T-B$ plane, which coincides well with the region where the THE appears. While the magnitude of THE is substantially larger under $P$ than in doped samples, the ratio $f$ of the effective field to its theoretical maximum remains comparable in both cases.

Acknowledgement The authors thank M. Hermele, D. 
Reznik, and J. A. Yang for enlightening discussions. This work is supported by the U.S. Department of Energy, Office of Basic Energy Sciences, Division of Materials Sciences and Engineering under Award ER 46797.

$\dagger$ Current Address: Department of Physics and Astronomy, University of California, Riverside CA

* Electronic address: minhyea.lee@colorado.edu

[1] S. Mühlbauer, B. Binz, F. Jonietz, C. Pfleiderer, A. Rosch, A. Neubauer, R. Georgii, and P. Bni, Science 323, 915 (2009).

[2] W. Münzer, A. Neubauer, T. Adams, S. Mühlbauer, C. Franz, F. Jonietz, R. Georgii, P. Böni, B. Pedersen, M. Schmidt, et al., Phys. Rev. B 81, 041203 (2010).

[3] X. Yu, N. Kanazawa, Y. Onose, K. Kimoto, W. Zhang, S. Ishiwata, Y. Matsui, and Y. Tokura, Nature materials 10, 106 (2010).

[4] S. Seki, X. Z. Yu, S. Ishiwata, and Y. Tokura, Science 336, 198 (2012).

[5] X. Z. Yu, B. Onose, Y., N. Kanazawa, J. H. Park, J. H. Han, Y. Matsui, N. Nagaosa, and Y. Tokura, Nature 465, 901 (2010).

[6] A. Bogdanov and D. Yablonskii, Zh. Eksp. Teor. Fiz 95, 182 (1989).

[7] T. Adams, A. Chacon, M. Wagner, A. Bauer, G. Brandl, B. Pedersen, H. Berger, P. Lemmens, and C. Pfleiderer, Phys. Rev. Lett. 108, 237204 (2012).

[8] T. Moriya, Phys. Rev. 120, 91 (1960).

[9] I. Dzyaloshinsky, Journal of Physics and Chemistry of Solids 4, 241 (1958), ISSN 0022-3697.

[10] C. Pfleiderer, T. Adams, A. Bauer, W. Biberacher, B. Binz, F. Birkelbach, P. Bni, C. Franz, R. Georgii, M. Janoschek, et al., Journal of Physics: Condensed Matter 22, 164207 (2010).

[11] P. Bruno, V. K. Dugaev, and M. Taillefumier, Phys. Rev. Lett. 93, 096806 (2004).

[12] T. Schulz, R. Ritz, A. Bauer, M. Halder, M. Wagner, C. Franz, C. Pfleiderer, K. Everschor, M. Garst, and A. Rosch, Nature Physics 8, 301 (2012).
[13] Y. Aharanov and D. Bohm, Phys. Rev. 115, 485 (1959).

[14] J. Ye, Y. B. Kim, A. J. Millis, B. I. Shraiman, P. Majumdar, and Z. Tešanović, Phys. Rev. Lett. 83, 3737 (1999).

[15] L. Ioffe, G. Lesovik, and A. Millis, Phys. Rev. Lett 77, 1584 (1996).

[16] N. Nagaosa, J. Sinova, S. Onoda, A. H. MacDonald, and N. P. Ong, Rev. Mod. Phys. 82, 1539 (2010).

[17] R. Ritz, M. Halder, C. Franz, A. Bauer, M. Wagner, R. Bamler, A. Rosch, and C. Pfleiderer, Phys. Rev. B 87, 134424 (2013).

[18] B. Binz and A. Vishwanath, Physica B: Condensed Matter 403, 1336 (2008).

[19] K. Koyama, T. Goto, T. Kanomata, and R. Note, Phys. Rev. B 62, 986 (2000).

[20] M. Lee, Y. Onose, Y. Tokura, and N. P. Ong, Phys. Rev. B 75, 172403 (2007).

[21] A. Neubauer, C. Pfleiderer, B. Binz, A. Rosch, R. Ritz, P. G. Niklowitz, and P. Böni, Phys. Rev. Lett. 102, 186602 (2009).

[22] S. V. Grigoriev, V. A. Dyadkin, E. V. Moskvin, D. Lamago, T. Wolf, H. Eckerlebe, and S. V. Maleyev, Phys. Rev. B 79, 144417 (2009).

[23] B. Fåk, R. A. Sadykov, J. Flouquet, and G. Lapertot, Journal of Physics: Condensed Matter 17, 1635 (2005).

[24] A. Neubauer, C. Pfleiderer, B. Binz, A. Rosch, R. Ritz, P. G. Niklowitz, and P. Böni, Phys. Rev. Lett. 110, 209902 (2013).

[25] M. Lee, W. Kang, Y. Onose, Y. Tokura, and N. P. Ong, Phys. Rev. Lett. 102, 186601 (2009).

[26] S. V. Grigoriev, E. V. Moskvin, V. A. Dyadkin, D. Lamago, T. Wolf, H. Eckerlebe, and S. V. Maleyev, Phys. Rev. B 83, 224411 (2011).

[27] A. Bauer, A. Neubauer, C. Franz, W. Münzer, M. Garst, and C. Pfleiderer, Phys. Rev. B 82, 064404 (2010).

[28] A. Aharoni, Journal of Applied Physics 83, 3432 (1998), ISSN 0021-8979.

[29] J. G. Checkelsky, M. Lee, E. Morosan, R. J. Cava, and N. P. Ong, Phys. Rev. B 77, 014433 (2008).

[30] Y. J. Uemura, T. Goko, I. M. Gat-Malureanu, J. P. Carlo, P. L. Russo, A. T. Savici, A. Aczel, G. J. MacDougall, J. A. Rodriguez, G. M. Luke, et al., Nature Physics 3, 29 (2006). 